\title{
Dimer-promoted fluorescence quenching of coralyne by binding to anionic polysaccharides
}

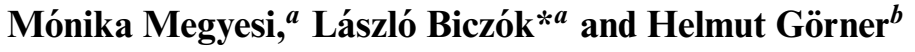 \\ Received 17th December 2008, Accepted 16th February 2009 \\ First published as an Advance Article on the web 26th February 2009 \\ DOI: 10.1039/b822649k
}

The effects of anionic chondroitin or dextran sulfates on the absorption and fluorescence properties of coralyne, a cationic benzo[c]phenanthridine type alkaloid, were studied in aqueous solution. The remarkably strong binding to both polysaccharides promotes the coralyne dimer formation, which was evidenced by the changes in the absorption and fluorescence spectra and the fluorescence decay. The extent of dimerization, induced by chondroitin, shows a significant $\mathrm{pH}$ dependence because the competitive protonation of the carboxylate moieties of the polymer chain decreases the number of binding sites. A larger molecular weight of dextran sulfate stabilizes the coralyne dimer more efficiently.

\section{Introduction}

The binding of oppositely charged species to water soluble polyelectrolytes has been extensively studied because this type of interaction plays a pivotal role in biological systems, biomedical applications, drug delivery and controlled release of biologically active compounds. ${ }^{1}$ Synthetic polyions act as model systems for the understanding of the often far more complex behaviour of the natural charged macromolecules such as proteins and DNA. ${ }^{2}$ Polyelectrolyte-dye complexes can self-aggregate to nanoparticles leading to amplified quenching of its fluorescence, which can be exploited in the design of sensitive optical sensors. ${ }^{3}$ Some effects of counter ions, temperature and the characteristics of the components were studied in polyelectrolyte-antidepressant drug complexes. ${ }^{4,5}$ To the best of our knowledge, no information is available on the binding of alkaloids to polyanions other than DNA.

In the present work, we focus on coralyne, a monocationic benzo[c]phenanthridine type alkaloid (Scheme 1), which intercalates in DNA, ${ }^{6}$ induces DNA aggregation or precipitation, ${ }^{7}$ stabilizes triple helices ${ }^{8}$ and alters the nucleic acid secondary structure. ${ }^{9}$ Its biological activity and interaction with polynucleotides have been recently reviewed. ${ }^{10}$ Spectrophotometric measurements demonstrated that it forms dimers and multiple aggregates in water. The increase of the ionic strength or the presence of small amount of DNA was found to promote the association. ${ }^{11}$ Garcia et al. revealed the effect of ethanol on the thermodynamics and kinetics of coralyne aggregation. ${ }^{12}$ Coralyne initiates very fast photochemical DNA damage by single-strand cleavage. Although the reaction mechanism is unknown, coralyne aggregates were suggested to serve as photosensitizers in this process. ${ }^{13}$ The fluorescence intensity alteration of coralyne-impregnated silica gel plates allows a sensitive quantitative detection of a wide number

${ }^{a}$ Chemical Research Center, Hungarian Academy of Sciences, P.O. Box 17, 1525, Budapest, Hungary.E-mail: biczok@chemres.hu

${ }^{b}$ Max-Planck-Institute for Bioinorganic Chemistry, P.O. Box 101365, D45413, Mülheim an der Ruhr, Germany. E-mail: goerner@mpi-muelheim. mpg.de of analytes by high-performance thin layer chromatographic techniques. ${ }^{14}$

Our main goal is to explore how the photophysical characteristics of coralyne change upon self-aggregation and association with negatively charged polysaccharides, such as a sodium salt of chondroitin-6-sulfate (CS) and two dextran sulfates. The former compound is a glycosaminoglycan, which is a major component of extracellular matrix and a widely used dietary supplement for treatment of osteoarthritis. It has an unbranched chain composed of about 100 alternating $N$-acetylgalactosamine and glucuronic acid moieties. Owing to the presence of the carboxylate and sulfate groups (Scheme 1), each repetitive disaccharide unit possesses two negative charges. Dextran sulfate salts having average molecular masses of 500000 and $5000 \mathrm{Da}$ (D500k and D5k) and branched structures were also employed as polyelectrolytes since side chains can induce a coil conformation. ${ }^{15}$ The repulsion of the negatively charged sulfate groups stabilizes the fully extended molecular structure in low ionic strength solution ${ }^{16}$ but condensation of counter ions can bring about conformational change. ${ }^{17}$ Dextran sulfate salts are known for their antithrombotic and antiviral activity ${ }^{18}$ and commonly used as both experimental and clinical laboratory reagents.

\section{Experimental}

Coralyne chloride (Acros Organics) was recrystallized from ethanol. Sodium salts of chondroitin-6-sulfate (BioChemika Fluka from shark cartilage) and dextran sulfates D500k and D5k (Fluka) were used as received. According to the specification of the manufacturer, dextran sulfates contain on average 2.3 sulfate groups per glucoside unit. For the calculation of the molar concentration, 396 and $503 \mathrm{Da}$ were used as the molecular mass of the sodium salt of dextran sulfate monomer and chondroitin6-sulfate disaccharide repeating units. The UV-visible absorption spectra were recorded on a Unicam UV 500 spectrophotometer. Corrected fluorescence spectra were obtained on a Jobin-Yvon Fluoromax-P photon-counting spectrofluorometer and the fluorescence yields $\left(\Phi_{\mathrm{F}}\right)$ were determined relative to that of quinine 
<smiles>COc1cc2cc3c4cc(OC)c(OC)cc4cc[n+]3c(C)c2cc1OC</smiles>

Coralyne cation

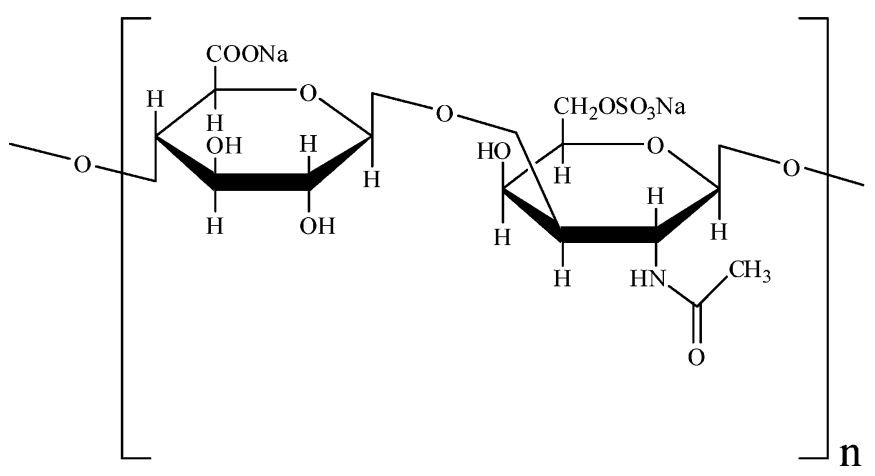

Chondroitin-6-sulfate sodium salt

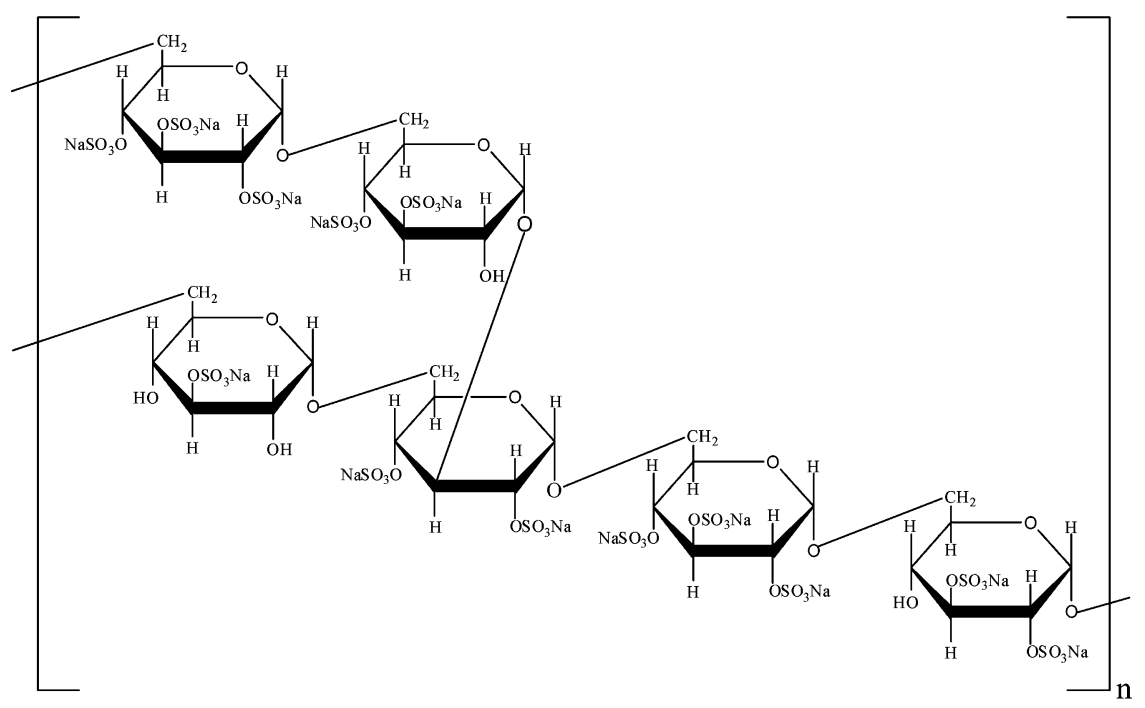

Dextran sulfate sodium salt

Scheme 1

sulfate in $0.5 \mathrm{M} \mathrm{H}_{2} \mathrm{SO}_{4}$ solution, for which a reference yield of $\Phi_{\mathrm{F}}=0.546$ was taken. ${ }^{19}$ Fluorescence decays were measured with the time-correlated single-photon-counting technique. A Picoquant diode laser (pulse duration $c a .70$ ps, wavelength $372 \mathrm{~nm}$ ) excited the samples, and the fluorescence decays were detected with a Hamamatsu R3809U-51 microchannel plate photomultiplier, which was connected to a Picoquant Timeharp 100 electronics (36 ps/channel time resolution). All experiments were carried out at $296 \mathrm{~K}$. Data were analyzed by a nonlinear least-squares deconvolution method using Picoquant FluoFit software.

\section{Results and discussion}

\section{Association in water}

As seen in Fig. 1, the spectral characteristics of coralyne depend significantly on the concentration. Wilson and coworkers have 

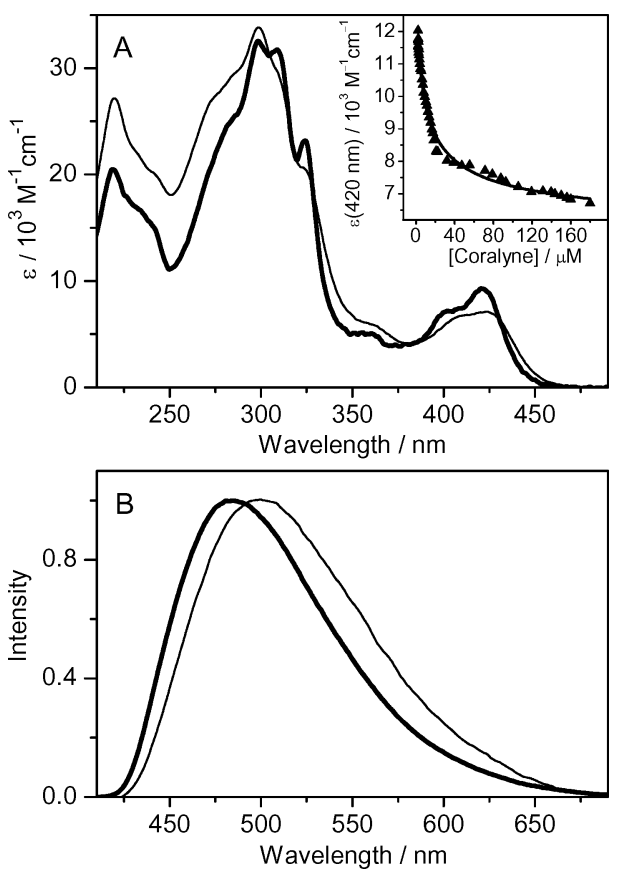

Fig. 1 Absorption (A) and fluorescence emission $\left(B, \lambda_{\text {exc }}=350 \mathrm{~nm}\right.$ ) spectra of 3.1 (thick line) and $40.2 \mu \mathrm{M}$ (thin line) coralyne in aqueous solution at $\mathrm{pH} 6$. The inset shows the apparent molar absorption coefficient at $420 \mathrm{~nm}$ as a function of the coralyne concentration. The line represents the best non-linear least-squares fit.

studied the coralyne absorption above $c a .350 \mathrm{~nm}$. They observed hypochromicity and a small red-shift of the lowest energy absorption band with increasing concentration. The spectral changes were attributed to dimerization for which $1.1 \times 10^{5} \mathrm{M}^{-1}$ equilibrium constant was calculated in neat water. ${ }^{11}$ Our results are in accordance with these findings, and an examination in a wider spectral domain showed that significant alterations also occur in the higher energy absorption bands. A recent paper, published during the preparation of our revised manuscript, also demonstrates the concentration dependence of the coralyne absorption..$^{12}$ As a representative example, the inset to Fig. 1 displays the variation of the apparent molar absorption coefficient at $420 \mathrm{~nm}$ with increasing total coralyne concentration $\left([\mathrm{C}]_{\mathrm{T}}\right)$. On the basis of the equilibrium constant of dimer (D) formation, $K=$ $[\mathrm{D}] /[\mathrm{M}]^{2}$, the fraction $(\alpha)$ of $[\mathrm{C}]_{\mathrm{T}}$ present as monomer $(\mathrm{M})$ is given by the following equation:

$$
\alpha=\frac{-1+\sqrt{1+8 K[\mathrm{C}]_{\mathrm{T}}}}{4 K[\mathrm{C}]_{\mathrm{T}}}
$$

The measured apparent molar absorption coefficient $(\varepsilon)$ is:

$$
\varepsilon=\varepsilon(\mathrm{M}) \alpha+\varepsilon(\mathrm{D})(1-\alpha) / 2
$$

where $\varepsilon(\mathrm{M})$ and $\varepsilon(\mathrm{D})$ represent the molar absorption coefficients for the monomer and dimer, respectively. The computed function, displayed in the inset to Fig. 1 as solid line, matches the experimental data well, and the parameters corresponding to the best fit are: $K=1.1 \times 10^{5} \mathrm{M}^{-1}, \varepsilon(\mathrm{M})=14100 \mathrm{M}^{-1} \mathrm{~cm}^{-1}$ and $\varepsilon(\mathrm{D})=$ $11200 \mathrm{M}^{-1} \mathrm{~cm}^{-1}$ at $420 \mathrm{~nm}$. NMR measurements at high $(5 \mathrm{mM})$ coralyne concentration by Garcia et al. provided a somewhat lower, $8693 \pm 102 \mathrm{M}^{-1}$ value for the self-aggregation constant. ${ }^{12}$
Under their experimental conditions not only dimer but also a significant amount of larger aggregates were formed. The results of NMR measurements implied that the monomer aggregation came about by stacking interactions, which involve mainly hydrophobic forces. ${ }^{12}$

The normalized fluorescence spectra (Fig. 1B) demonstrate a bathochromic displacement and a slight broadening of the emission band in concentrated solution. The fluorescence maximum is plotted as a function of the concentration in Fig. 2A. The approximately $16 \mathrm{~nm}$ red-shift of the peak is accompanied by the diminution of the fluorescence quantum yield from 0.34 to 0.20 on going from 3 to $40 \mu \mathrm{M}$ coralyne solution (Fig. 2B). Using the $K=$ $1.1 \times 10^{5} \mathrm{M}^{-1}$ value for the equilibrium constant of dimerization, we can calculate that from 69 to $28 \%$ of coralyne is present as monomer in this concentration range. Thus, the decline of $\Phi_{\mathrm{F}}$ is due to the weaker emission of the dimer.

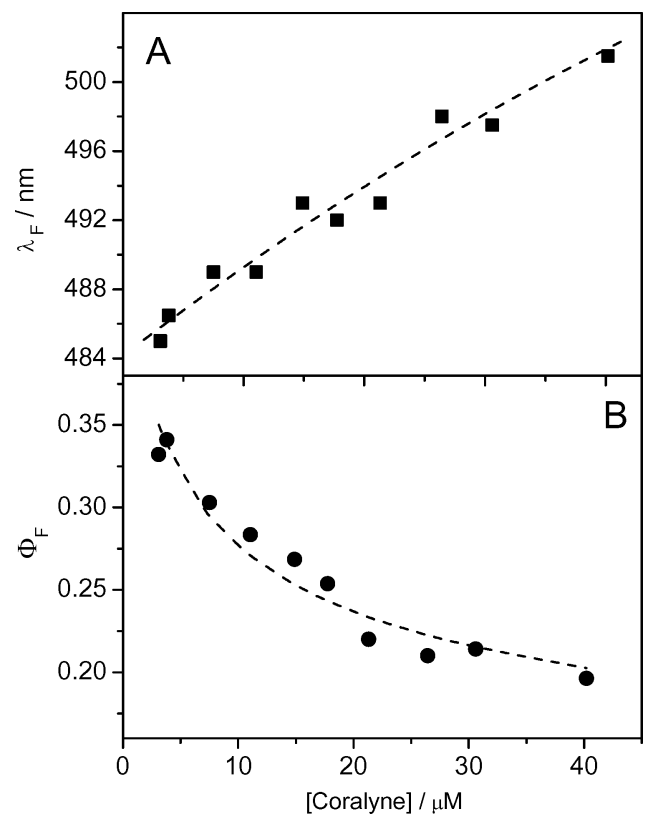

Fig. 2 Coralyne concentration dependence of the fluorescence maximum (A) and the fluorescence quantum yield (B) in aqueous solution at $\mathrm{pH} 6$.

Time-resolved fluorescence measurements showed an exponential decay with a lifetime of $\tau_{1}=9.7 \mathrm{~ns}$ below $0.04 \mu \mathrm{M}$ coralyne concentration. When the concentration was raised, a long-lived fluorescence component with a lifetime of $\tau_{2}=40.7 \mathrm{~ns}$ appeared with gradually increasing amplitude due to dimerization. Both decay parameters were constant within the limits of experimental errors in the whole concentration domain indicating that no higher aggregates were produced. The longer lifetime and lower $\Phi_{\mathrm{F}}$ of the dimer compared to the monomer suggest that association slows down both the radiative and the radiationless deactivations of the singlet-excited state.

\section{Effects of CS in neutral solution}

The effect of polysaccharides in aqueous solution was examined for $2.7 \mu \mathrm{M}$ coralyne, where the majority of the alkaloid molecules are present as monomers. In order to eliminate the effect of polydispersity, the amount of polyelectrolyte is given as the concentration of the disaccharide repeat unit. Fig. 3 depicts the change of 


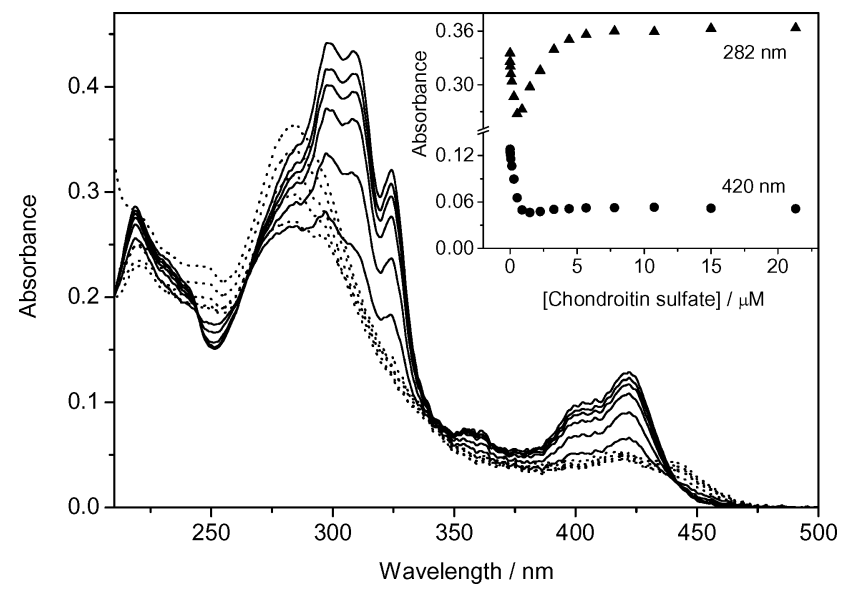

Fig. 3 Absorption spectra of $2.7 \mu \mathrm{M}$ coralyne in aqueous solution at $\mathrm{pH} 6$ and in the presence of $0,0.028,0.066,0.14,0.29,0.54 \mu \mathrm{M}$ (solid line) and $0.90,1.5,2.2,3.3,21 \mu \mathrm{M}$ (dotted line) CS (optical path is $5 \mathrm{~cm}$ ). Inset presents the absorbance change at $282(\boldsymbol{\Delta})$ and $420 \mathrm{~nm}(\boldsymbol{\bullet})$ as a function of the CS concentration.

the absorption spectrum upon addition of gradually increasing amounts of CS and the inset illustrates the absorbance variation at 282 and $420 \mathrm{~nm}$. In the $0-0.54 \mu \mathrm{M}$ CS concentration domain marked hypochromicity is observed and the isosbestic points appearing at 245, 265 and $442 \mathrm{~nm}$ indicate $1: 1$ complexation of coralyne to the CS repeating sequence. Further augmentation of CS concentration brings about a bathochromic displacement of the first absorption band and a concomitant rise of a new absorption band with a maximum at $284 \mathrm{~nm}$. This suggests that the binding of a second coralyne molecule to the disaccharide segment facilitates dimer formation. The absorption spectrum of CS-bound and free dimers are displayed in Fig. 4. For the sake of better comparison, the two spectra are normalized at the maximum of the most intense band. On the basis of eqn (1) and $K=1.1 \times$ $10^{5} \mathrm{M}^{-1}, 81.4 \%$ of coralyne is dimerized in $107 \mu \mathrm{M}$ aqueous solution. When CS induces aggregation, a somewhat different spectrum is obtained indicating that the association with CS probably modifies the structure of the dimer and the association of more than two coralyne to the disaccharide unit of CS cannot be excluded. The charge recombination upon association with the negatively charged CS decreases the electrostatic repulsion

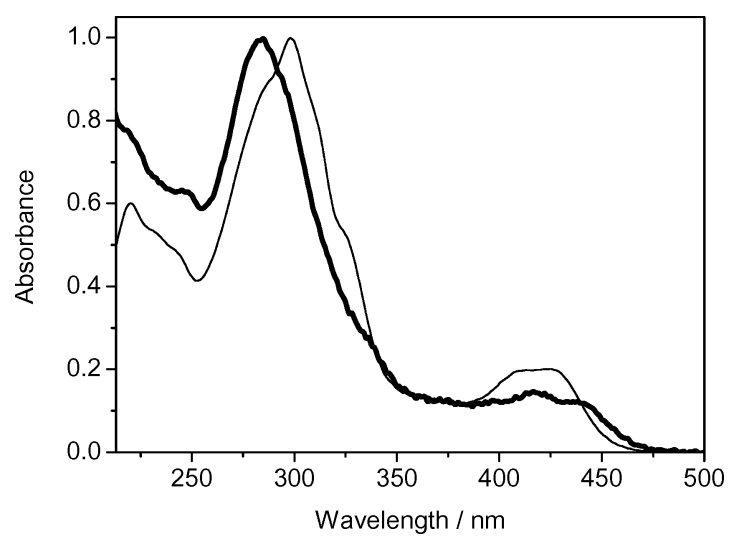

Fig. 4 Normalized absorption spectra of $107 \mu \mathrm{M}$ coralyne (thin line) and $2.7 \mu \mathrm{M}$ coralyne $+21 \mu \mathrm{M} \mathrm{CS}$ (thick line) in water at $\mathrm{pH} 6$. between the bound coralyne cations and the conformation change of the polymer may bring them close enough to interact with each other. Probably hydrophobic forces and the $\pi-\pi$ interaction between the flat moieties facilitate the dimerization.

As shown in Fig. 5, addition of even a small amount $(<0.7 \mu \mathrm{M})$ of CS brings about a significant fluorescence quenching but only a negligible shift of the fluorescence maximum is observed in such diluted solutions. In contrast, a moderate fluorescence intensity diminution and a marked red-shift of the fluorescence maximum is seen in the $0.7-1.6 \mu \mathrm{M}$ CS concentration range but these changes level off and the spectral characteristics do not alter up to a CS concentration of about $0.1 \mathrm{mM}$. In more concentrated polymer solutions, fluorescence enhancement and a blue-shift of the emission peak were detected.
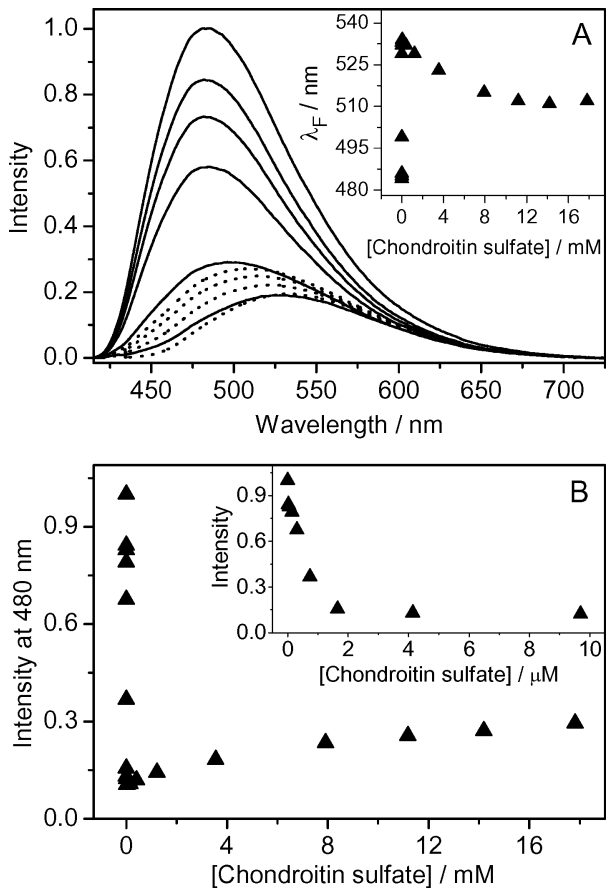

Fig. 5 (A) Fluorescence spectra of $3.16 \mu \mathrm{M}$ coralyne aqueous solution at $\mathrm{pH} 6$ on addition of $0,0.022,0.14,0.31,0.74,1.6 \mu \mathrm{M}$ (solid line), $0.14,3.6,7.9,14 \mathrm{mM}$ (dotted line) CS, excitation at $372 \mathrm{~nm}$. Inset shows the fluorescence maximum $v s$. chondroitin sulfate concentration. (B) The fluorescence intensity at $480 \mathrm{~nm}$ corrected by the change of absorbance at the excitation wavelength $(372 \mathrm{~nm})$ plotted as a function of $\mathrm{CS}$ concentration at $\mathrm{pH}$ 6. Inset represents the low concentration domain.

In order to gain deeper insight into the origin of the significant alteration of the fluorescent behaviour, time-resolved measurements were performed in $3.16 \mu \mathrm{M}$ coralyne solution. Below $0.7 \mu \mathrm{M}$ CS concentration, exponential fluorescence decays with an invariable lifetime were observed. This implies that the $1: 1$ complexation of coralyne to the disaccharide unit of CS does not alter the fluorescence decay kinetics. However, the considerable reduction of the fluorescence intensity (Fig. 5) indicates that the radiative rate constant decreases upon $1: 1$ binding. The slowdown of the radiative deactivation from the singlet excited state upon $1: 1$ complexation is probably compensated by the acceleration of the radiationless energy dissipation processes.

In the presence of $0.7 \mu \mathrm{M} \mathrm{CS}$, a long-lived fluorescence component with a constant $61 \mathrm{~ns}$ lifetime emerged, whose fractional 
amplitude $\left(a_{2} /\left(a_{1}+a_{2}\right)\right)$ grew up to $54 \mu \mathrm{M} \mathrm{CS}$ concentration, and thereafter declined (Fig. 6). This emission is attributed to interacting polymer-bound coralyne. Each disaccharide unit has two anionic substituents, a carboxylate and a sulfate groups, providing links to coralyne cations. The saturation of the anionic binding sites by the oppositely charged alkaloid eliminates the electrostatic repulsion among the polyelectrolyte segments thereby allowing larger flexibility in the polymer chain. The more facile conformational movement of the polysaccharide backbone promotes the interaction between the bound alkaloid molecules. The diminution of the $a_{2} /\left(a_{1}+a_{2}\right)$ values at CS concentration above $54 \mu \mathrm{M}$ reflects the reduced mobility of the polymer chain and the lesser chance for binding to two neighboring sites, where guests can interact. The fractional amplitude of the long-lived fluorescence does not vanish completely at high CS concentration in water, indicating that the polyelectrolyte-bound coralyne can dimerize more efficiently than the free coralyne. The fluorescence enhancement and a blue-shift of the emission at high CS concentration are due to the decreasing extent of dimerization.

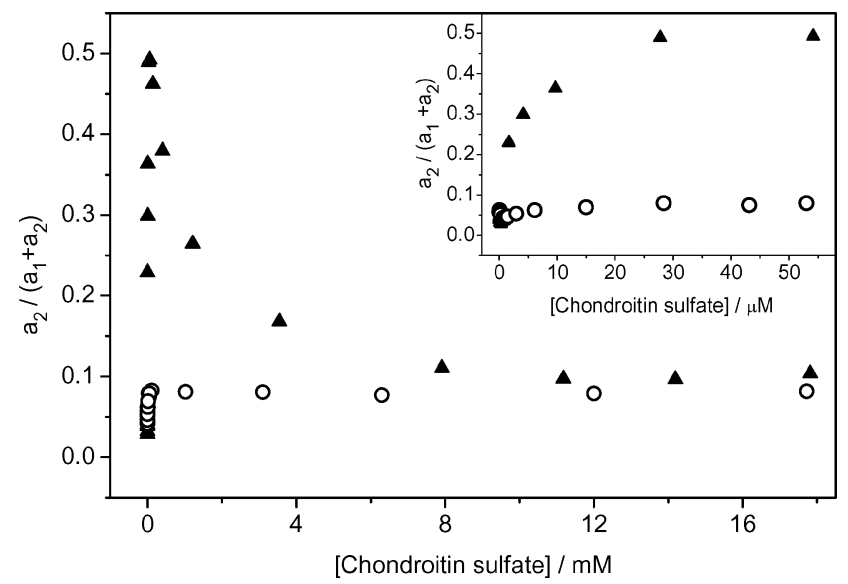

Fig. 6 Fractional amplitude of the long-lived fluorescence component detected at $490 \mathrm{~nm}$ as a function of the CS concentration in $3.16 \mu \mathrm{M}$ coralyne solution. Inset represents the zoomed view of the initial part. Triangles and open circles represent the results at $\mathrm{pH} 6$ and 2, respectively.

\section{Effect of $\mathrm{CS}$ at $\mathrm{pH} 2$}

In acidic solution, CS has a different effect on the fluorescence of coralyne than in neutral conditions. As seen in the inset to Fig. 7B, the intensity diminution at $\mathrm{pH} 2$ levels off at about $3 \mu \mathrm{M} \mathrm{CS}$, which corresponds to $1: 1$ complexation stoichiometry. The $2: 1$ association with a repeating unit is hindered because at low $\mathrm{pH}$ the carboxylate groups of the polymer are blocked by protonation. Above $3 \mu \mathrm{M}$ CS concentration, a weak fluorescence component with $\tau_{2}=55 \mathrm{~ns}$ appears but its fractional amplitude $\left(a_{2} /\left(a_{1}+a_{2}\right)\right)$ does not exceed $8 \%$. This indicates the small extent of coralyne dimer formation when only a single binding site is available in the repeat unit of the polymer. The remarkable difference in the CS concentration dependence of $a_{2} /\left(a_{1}+a_{2}\right)$ at $\mathrm{pH} 2$ and 6 is displayed in Fig. 6. The $a_{2} /\left(a_{1}+a_{2}\right)$ fractional amplitudes at $\mathrm{pH} 6$ approach the value obtained in acidic solution when the large excess of CS provides little chance for binding two coralyne molecules to the neighboring sites of the polysaccharide. The shorter-lived fluorescence components have the same lifetime (9.7 ns) irrespective of the $\mathrm{pH}$ and $\mathrm{CS}$ concentration.
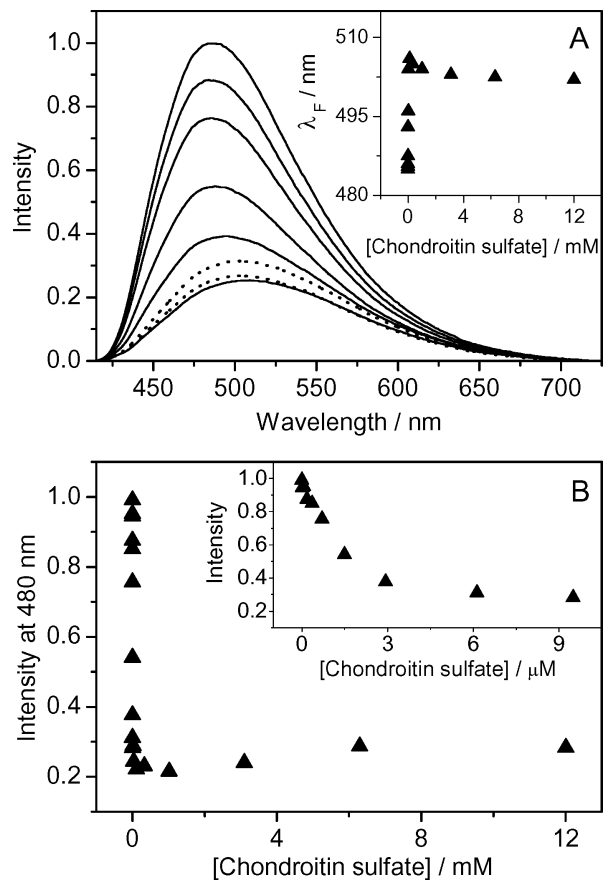

Fig. 7 (A) Fluorescence spectra of $3.13 \mu \mathrm{M}$ coralyne in aqueous solution at $\mathrm{pH} 2$ in the presence of $0,0.18,0.71,1.5,2.9,120 \mu \mathrm{M}$ (solid line), 3.1 and $12 \mathrm{mM}$ (dotted line) CS, excitation at $372 \mathrm{~nm}$. Inset shows the variation of the fluorescence maximum. (B) Concentration dependence of the fluorescence intensity at $480 \mathrm{~nm}$ at $\mathrm{pH} 2$. Inset represents the low concentration region.

\section{Effect of dextran sulfate salts in neutral solution}

In order to reveal how the replacement of carboxylates by sulfate moieties on the polysaccharide chain influences the binding of coralyne, dextran sodium sulfate salts were used as additives. The molecular weight of these substances was changed from 5000 to $500000 \mathrm{Da}$ in order to unravel the effect of the chain length on the association. Addition of dextran sulfate leads to analogous alteration in the absorption and fluorescence properties as described for CS (vide supra) but the extent of change depends on the degree of polymerization. The inset to Fig. 8 illustrates that D500k induces coralyne dimerization more efficiently than D5k. The repeat unit concentration, where the factional amplitude of coralyne dimer fluorescence $\left(a_{2} /\left(a_{1}+a_{2}\right)\right)$ reaches a limiting value, is lower for D500k than for D5k. In the presence of the former polymer, the $a_{2} /\left(a_{1}+a_{2}\right)$ values diminish slower above $0.5 \mathrm{mM}$ repeat unit concentration. A similar behavior appears for the fluorescence maximum (Fig. 8B). It is known that the degree of branching of dextran sulfates increases with the molecular weight. ${ }^{17}$ When the polymer has more side chains and the main chain is longer, encounters of the bound coralyne molecules are more likely and a less polar microenvironment can be developed. Both effects may contribute to the larger stability of the D500k bound dimer.

\section{Conclusions}

Coralyne in neutral aqueous solution exhibits a large affinity to anionic polysaccharides due to electrostatic and hydrophobic interactions. The binding promotes coralyne dimerization even in 


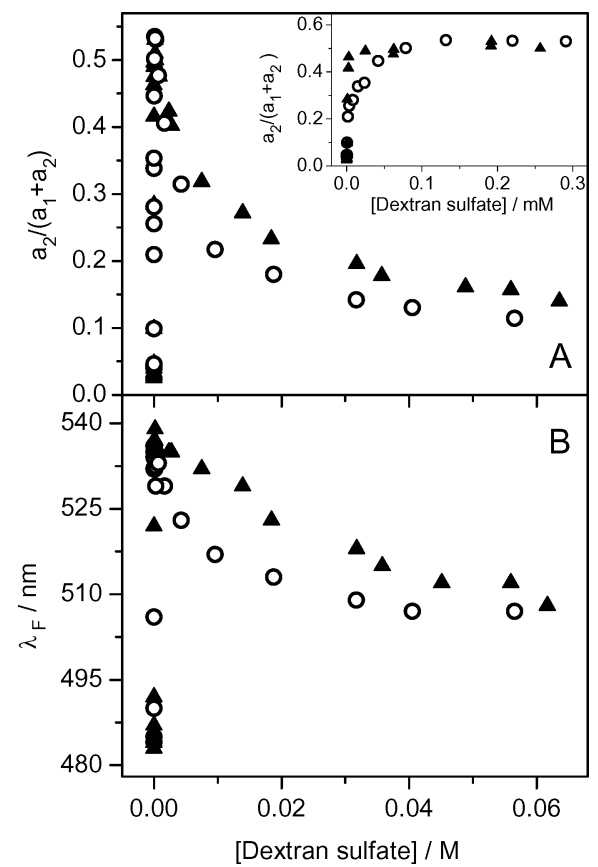

Fig. 8 Dextran sulfate sodium salt concentration dependence of the fractional amplitude of the long-lived fluorescence decay component (A) and the fluorescence maximum (B) of $3.2 \mu \mathrm{M}$ coralyne in aqueous solution at $\mathrm{pH}$ 6. Inset shows a zoomed view. Open circles and filled triangles represent the results obtained with a molecular weight of 5 and $500 \mathrm{kDa}$, respectively.

the presence of a polymeric repeat unit in large concentration. Chondroitin and dextran sulfates induce similar changes in the absorption and fluorescence characteristics of coralyne. The stability of the polymer-bound coralyne dimer is significantly diminished by the protonation of the carboxylate groups of chondroitin, whereas the change is smaller when the molecular weight of dextran is increased by a factor 100 .

\section{Acknowledgements}

The authors very much appreciate the support of this work by the Hungarian Scientific Research Fund (OTKA, Grant K75015) and the bilateral program between the Deutsche Forschungsgemeinschaft and the Hungarian Academy of Sciences.

\section{References}

1 (a) B. G. De Geest, N. N. Sanders, G. B. Sukhorukov, J. Demeester and S. C. De Smedt, Release mechanisms for polyelectrolyte capsules, Chem. Soc. Rev., 2007, 36, 636; (b) M. C. Bonferoni, G. Sandri, E. Gavini, S. Rossi, F. Ferrari and C. Caramella, Microparticle systems based on polymer-drug interaction for ocular delivery of ciprofloxacinI. In vitro characterization, J. Drug Delivery Sci. Technol., 2007, $17,57$.

2 U. Scheler, S. Schwarz, M. Müller, R. Farinato and P. Dubin, Advances in Polyelectrolyte Research, J. Phys. Chem. B, 2007, 111, 8343.

3 J. Chen, W. F. Dong, H. Möhwald and R. Krastev, Amplified fluorescence quenching of self-assembled polyelectrolyte-dye nanoparticles in aqueous solution, Chem. Mater., 2008, 20, 1664.
4 B. Persson, A. Hugerth, N. Caram-Lelham and L. O. Sundelöf, Dextran sulfate-amphiphile interaction; Effect of polyelectrolyte charge density and amphiphile hydrophobicity, Langmuir, 2000, 16, 313.

$5 \mathrm{~S}$. K. Singh and N. Caram-Lelham, Thermodynamics of $\kappa-$ carrageenan-amphiphilic drug interaction as influenced by specific counterions and temperature: A microcalorimetric and viscometric study, J. Colloid Interface Sci., 1998, 203, 430.

6 (a) W. D. Wilson, A. N. Gough, J. J. Doyle and M. W. Davidson, Coralyne-intercalation with DNA as a possible mechanism of antileukemic action, J. Med. Chem., 1976, 19, 1261; (b) S. Pal, S. Das, G. S. Kumar and M. Maiti, Antitumour agent coralyne: A guaninecytosine specific DNA-binding alkaloid, Curr. Sci., 1998, 75, 496; (c) K. Bhadra, M. Maiti and G. S. Kumar, Thermodynamics of the binding of cytotoxic protoberberine molecule coralyne to deoxyribonucleic acids, Biochim. Biophys. Acta, 2008, 1780, 298

7 Z. Taira, M. Matsumoto, S. Ishida, T. Ichikawa and Y. Sakiya, Aggregation of DNA enhanced by the protoberberine alkaloids, coralyne and berberine, Chem. Pharm. Bull., 1994, 42, 1556.

8 (a) J. S. Lee, L. J. P. Latimer and K. J. Hampel, Coralyne binds tightly to both T.A.T-containing and C.G.C. $\mathrm{C}^{+}$-containing DNA triplexes, Biochemistry, 1993, 32, 5591; (b) M. Polak and N. Hud, Complete disproportionation of duplex poly $(\mathrm{dT}) \cdot \operatorname{poly}(\mathrm{dA})$ into triplex poly(dT) $\operatorname{poly}(\mathrm{dA}) \cdot \operatorname{poly}(\mathrm{dT})$ and poly(dA) by coralyne, Nucleic Acids Res., 2002, 30, 983; (c) A. A. Moraru-Allen, S. Cassidy, J. L. A. Alvarez, K. R. Fox, T. Brown and A. N. Lane, Coralyne has a preference for intercalation between T.A.T triples in intramolecular DNA triple helices, Nucleic Acids Res., 1997, 25, 1890; (d) S. S. Jain, M. Polak and N. V. Hud, Controlling nucleic acid secondary structure by intercalation: Effects of DNA strand length on coralyne-driven duplex disproportionation, Nucleic Acids Res., 2003, 31, 4608.

9 (a) Ö. Persil, C. T. Santai, S. S. Jain and N. V. Hud, Assembly of an antiparallel homo-adenine DNA duplex by small-molecule binding, J. Am. Chem. Soc., 2004, 126, 8644; (b) F. Xing, G. Song, J. Ren, J. B. Chaires and X. Qu, Molecular recognition of nucleic acids: Coralyne binds strongly to poly(A), FEBS Lett., 2005, 579, 5035; (c) P. Giri and G. S. Kumar, Self-structure induction in single stranded poly(A) by small molecules: Studies on DNA intercalators, partial intercalators and groove binding molecules, Arch. Biochem. Biophys., 2008, 474, 183.

10 H. Ihmels, K. Faulhaber, D. Vedaldi, F. Dall'Acqua and G. Viola, Intercalation of organic dye molecules into double-stranded DNA. Part, 2: The annelated quinolizinium ion as a structural motif in DNA intercalators, Photochem. Photobiol., 2005, 81, 1107.

11 A. N. Gough, R. L. Jones and W. D. Wilson, Dimerization of coralyne and its propyl analog and their association with DNA, J. Med. Chem., 1979, 22, 1551 .

12 B. García, S. Ibeas, R. Ruiz, J. M. Leal, T. Biver, A. Boggioni, F. Secco and M. Venturini, Solvent effects on the thermodynamics and kinetics of coralyne self-aggregation, J. Phys. Chem. B, 2009, 113, 188.

$13 \mathrm{H}$. Ihmels and A. Salbach, Efficient photoinduced DNA damage by coralyne, Photochem. Photobiol., 2006, 82, 1572.

14 E. Mateos, V. L. Cebolla, L. Membrado, J. Vela, E. M. Gálvez, M. Matt and F. P. Cossio, Coralyne cation, a fluorescent probe for general detection in planar chromatography, J. Chromatogr. A, 2007, 1146, 251.

15 P. Beyer and E. Nordmeier, Some phenomena of counterion condensation on dextran sulfate, Eur. Polym. J., 1995, 31, 1031.

16 A. Katchalski, Polyelectrolytes and their biological interactions, Biophys. J., 1964, 4, 9

17 C. Schatz, J. M. Lucas, C. Viton, A. Alain, Domard, C. Pichot and T. Delair, Formation and properties of positively charged colloids based on polyelectrolyte complexes of biopolymers, Langmuir, 2004, 20, 7766.

18 (a) H. Mitsuya, D. J. Looney, S. Kuno, R. Ueno, F. Wong-Staal and S. Broder, Dextran sulfate suppression of viruses in the HIV familyInhibition of virion binding to CD4+ cells, Science, 1988, 240, 646; (b) M. Baba, R. Pauwels, J. Balzarini, J. Arnout, J. Desmyter and E. De, Clercq, Mechanism of inhibitory effect of dextran sulfate and heparin on replication of human immunodeficiency virus in vitro, Proc. Natl. Acad. Sci. U. S. A., 1988, 85, 6132.

19 W. H. Melhuish, Quantum efficiencies of fluorescence of organic substances: effect of solvent and concentration of the fluorescent solute, J. Am. Chem. Soc., 1961, 65, 229. 